\title{
Catalytic reduction of methylene blue and Congo red dyes using green synthesized gold nanoparticles capped by salmalia malabarica gum
}

\author{
Bhagavanth Reddy Ganapuram ${ }^{1} \cdot$ Madhusudhan Alle $^{1} \cdot$ Ramakrishna Dadigala $^{1} \cdot$ \\ Ayodhya Dasari $^{1} \cdot$ Venkatesham Maragoni $^{1} \cdot$ Veerabhadram Guttena $^{1}$
}

Received: 4 February 2015/Accepted: 17 August 2015/Published online: 29 August 2015

(C) The Author(s) 2015. This article is published with open access at Springerlink.com

\begin{abstract}
Stable gold nanoparticles (AuNPs) were synthesized using salmalia malabarica gum as both reducing and capping agent. It is a simple and eco-friendly green synthesis. The successful formation of AuNPs was confirmed by UV-visible spectroscopy (UV-Vis), Fourier transform infrared spectroscopy (FTIR), X-ray powder diffraction and transmission electron microscopy (TEM). The synthesized AuNPs were characterized by a peak at $520-535 \mathrm{~nm}$ in the UV-Vis spectrum. The X-ray diffraction studies indicated that the resulting AuNPs were highly crystalline with face-centred cubic geometry. TEM studies showed that the average particle size of the synthesized AuNPs was $12 \pm 2 \mathrm{~nm}$. FTIR analysis revealed that $-\mathrm{OH}$ groups present in the gum matrix might be responsible for the reduction of $\mathrm{Au}^{+3}$ into AuNPs. The synthesized AuNPs exhibited good catalytic properties in the reduction of methylene blue and Congo red.
\end{abstract}

Keywords Gold nanoparticles - Salmalia malabarica gum $\cdot$ Methylene blue $\cdot$ Congo red

\section{Introduction}

Metal nanoparticles such as platinum, gold, silver, etc., have been playing a significant role in the fields of biomedical, environmental, pharmaceutical, cosmetic, electronics and energy [1-3]. Among the noble metal nanoparticles, gold nanoparticles (AuNPs) show

Veerabhadram Guttena gvbhadram@gmail.com

1 Department of Chemistry, University College of Science, Osmania University, Hyderabad, Telangana 500007, India distinguished surface plasmon resonance (SPR) absorption properties which are strongly related to their size, shape and interparticle distance [4]. AuNPs find applications in various fields viz., manufacture of optical devices, surfaceenhanced Raman scattering, catalysis, colorimetric sensors, drug delivery, bioimaging [5-7] and so on.

Organic dyes are widely used in many industries such as textile, paper, pharmaceutical and food industries $[8,9]$. But, the excessive use of organic dyes leads to the environmental pollution that occurs from their undesirability, high visibility, recalcitrance and waste water has been a major concern for a long time. For that reason, the control of industrial effluents is an indispensable job which helps in the creation of a harmless and clean environment. Methylene blue (MB) and Congo red (CR) are cationic and anionic dyes, respectively [10]. These dyes are used extensively in textile, paper, rubber and plastic industries and cause serious ecological damage to the environment if they are discharged without proper action. Thus, the development of a simple method for the efficient degradation of dyes has gained greater significance. Due to their relatively large surface-to-volume ratios, metal nanoparticles show enhanced catalytic activity for the degradation of organic dyes [11].

There are several methods synthesize well-defined AuNPs such as chemical reduction, electrochemical, photochemical and sonochemical [12, 13], etc. But, these methods are harmful as they usually require the use of toxic chemicals which lead to the environmental toxicity or biological hazards. To prevent the negative impacts of the chemical reduction methods, researchers were interested to integrate "green chemistry" synthesis of nano materials using plant extracts, bio surfactants, etc., in aqueous medium [14]. Green synthesis of AuNPs has been reported using a variety of polysaccharides, including acacia nilotica leaf extract, xanthan gum, gellan gum, etc. [2, 15]. 
Here, green synthesis of AuNPs is attempted using salmalia malabarica gum (SMG) as reducing and stabilizing agent. SMG is a naturally occurring polysaccharide gum extracted from the plant Bombax ceiba, a native tree of India. The complete hydrolysis of gum has revealed that it contains a mixture of various sugars such as D-galacturonic acid, D-galactose, L-arabinose [16]. This gum is used in traditional ayurvedic and unani medical preparations for the treatment of anti-inflammatory, hepato-protective, hypotensive ailments and as an antioxidant and is also used for the treatment of asthma and diarrhoea [17, 18].

The present study reports the synthesis of AuNPs with salmalia malabarica gum acting as the reducing and stabilization agent. The synthesized nanoparticles were characterized by UV-visible spectroscopy (UV-Vis), Fourier transform infrared spectroscopy (FTIR), X-ray powder diffraction (XRD) and transmission electron microscopy (TEM) techniques. These AuNPs were also studied for their applications as a catalyst in the reduction of $\mathrm{MB}$ and $\mathrm{CR}$ in the presence of $\mathrm{NaBH}_{4}$ in water medium using UVVis spectrometry.

\section{Experimental section}

\section{Materials}

Chloroauric acid $\left(\mathrm{HAuCl}_{4} \cdot 3 \mathrm{H}_{2} \mathrm{O}\right)$ was purchased from Sigma Aldrich, Mumbai, India, and sodium borohydride $\left(\mathrm{NaBH}_{4}\right), \mathrm{MB}$ and $\mathrm{CR}$ were procured from Himedia Laboratories, Mumbai, India. Salmalia malabarica gum was obtained from Girijan Cooperative Society, Hyderabad, India. All the solutions were prepared in double distilled water.

\section{Synthetic procedure for gold nanoparticles}

All the glass ware was washed thoroughly with aquaregia to avoid any residual chemical contamination carried along with glassware. In a typical experiment, $1 \mathrm{~mL}$ of $1 \mathrm{mM}$ $\mathrm{HAuCl}_{4} \cdot 3 \mathrm{H}_{2} \mathrm{O}$ and $3 \mathrm{~mL}$ of $1 \%$ gum solution were added into a boiling tube for the synthesis of SMG capped AuNPs. The mixture was subjected to autoclaving at $121{ }^{\circ} \mathrm{C}$ and $15 \mathrm{psi}$ for $15 \mathrm{~min}$. The colour of the resultant solution was changed from pale yellow to red indicating the formation of the AuNPs from $\mathrm{Au}^{+3}$. The synthesis of nano particles was further confirmed by UV-Vis, FTIR, TEM and XRD techniques.

\section{Characterization techniques}

The resulting SMG capped AuNPs solution was analysed by UV-Vis absorption spectrophotometer (Model:
Shimadzu UV-Vis 3600, Shimadzu Corporation, Japan) in the range of 200-800 $\mathrm{nm}$. FTIR analysis was carried out on the aqueous solution of synthesized AuNPs using FTIR Spectrophotometer (Model: IRAffinity-1, Shimadzu Corporation, Japan) in the scanning range of $650-4000 \mathrm{~cm}^{-1}$. XRD analysis was conducted on a Rigaku-Miniflex method with Cuk $\alpha$ radiation. TEM analysis was performed using a transmission electron microscope (Model: 1200EX, JEOL Ltd., Japan).The presence of elemental gold was determined using a scanning electron microscope (Model: EDX Zeiss Evo 50, Carl Zeiss AG, Germany). The samples were dried at room temperature and then analysed for composition of the synthesized NPs.

\section{Procedure for reduction of dyes}

The reduction of $\mathrm{MB}$ and $\mathrm{CR}$ using sodium borohydride in the presence of AuNPs was carried out to demonstrate the catalytic activity of the prepared AuNPs. $1 \mathrm{~mL}$ of $10 \mathrm{mM}$ sodium borohydride solution was mixed with $1.5 \mathrm{~mL}$ of $1 \mathrm{mM} \mathrm{MB}$ and the mixture was made up to $10 \mathrm{~mL}$ using double-distilled water and then stirred for $5 \mathrm{~min} .1 \mathrm{~mL}$ of $10 \mathrm{mM} \mathrm{NaBH}_{4}$ solution was mixed with $1.5 \mathrm{~mL}$ of $1 \mathrm{mM}$ $\mathrm{CR}$, and the solution mixture was made up to $10 \mathrm{~mL}$ using doubled distilled water and then stirring was continued for $5 \mathrm{~min}$. To both these solutions, sufficient quantities of synthesized AuNPs were added separately and the UV-Vis spectra were recorded at regular intervals of time.

\section{Results and discussion}

\section{UV-Vis spectroscopy}

The UV-Vis spectroscopy is one of the most important and widely used simple and sensitive technique for determining the formation as well as size of the metal nanoparticles [19]. UV-Vis spectra of the AuNPs are presented in Fig. 1. The absorbance maximum was observed in the range of $523-535 \mathrm{~nm}$, which is characteristic of gold surface plasmon resonance (SPR). To optimize the synthesis of nanoparticles, the influence of parameters such as concentration of gum and concentration of chloroauric acid was studied. Figure 1 shows the UV-Vis spectra of the synthesized AuNPs with different concentrations of gum (0.1-1\%) with $1 \mathrm{mM} \mathrm{HAuCl} \mathrm{H}_{4}$ and $15 \mathrm{~min}$ of autoclaving time. After autoclaving, the reaction mixture with a red colour was observed from which it is evident that the AuNPs were formed by the reduction of chloroauric acid with gum. It also reveals that the formation of nanoparticles increases with increasing concentration of gum. Figure 2 shows the UV-Vis spectra of the synthesized AuNPs at different concentrations of chloroauric acid (0.1-1 mM) 


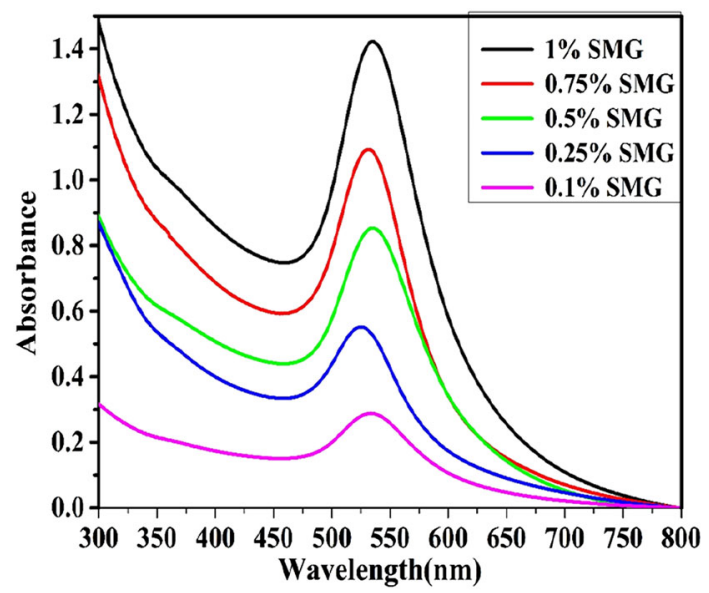

Fig. 1 UV-Vis absorption spectra of gold colloid suspensions showing different concentrations of gum SMG solutions at $1 \mathrm{mM}$ $\mathrm{HAuCl}_{4}$ concentration

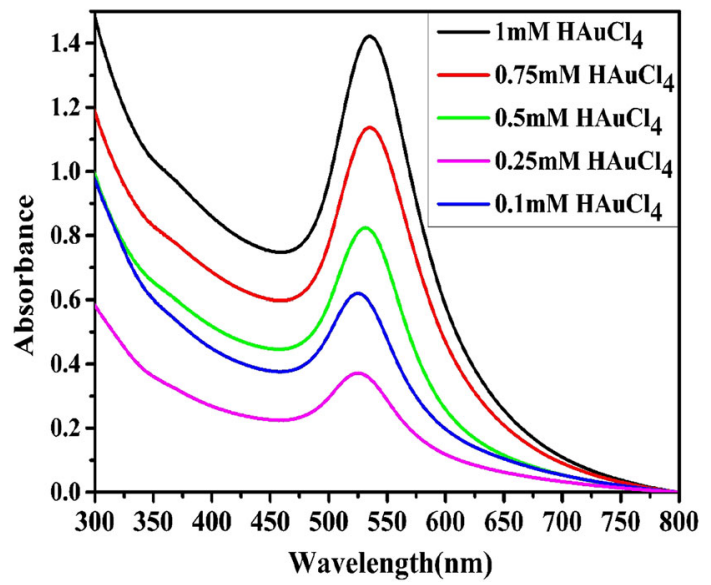

Fig. 2 UV-Vis spectra of the AuNPs obtained at various concentrations of $\mathrm{HAuCl}_{4}$ solutions and at $1 \%$ gum acacia concentration

containing $1 \%$ of gum with an autoclaving time of $15 \mathrm{~min}$ which demonstrates that the formation of nanoparticles increases with increase in the concentration of chloroauric acid.

\section{FTIR}

The identification of the possible functional groups involved in the reduction and the stabilization of greensynthesized AuNPs can be achieved by the FTIR spectroscopy. The major frequencies that are found in the FTIR spectrum of SMG are 3421, 2929, 2125, 1729, 1629, 1427 , 1240 and $1017 \mathrm{~cm}^{-1}$ (Fig. 3a). The broad band peak observed at $3421 \mathrm{~cm}^{-1}$ could be assigned to stretching vibrations of $-\mathrm{OH}$ groups in gum. The bands at $2929 \mathrm{~cm}^{-1}$ correspond to asymmetric stretching vibrations of methylene group. The broad band at $1729 \mathrm{~cm}^{-1}$ could be

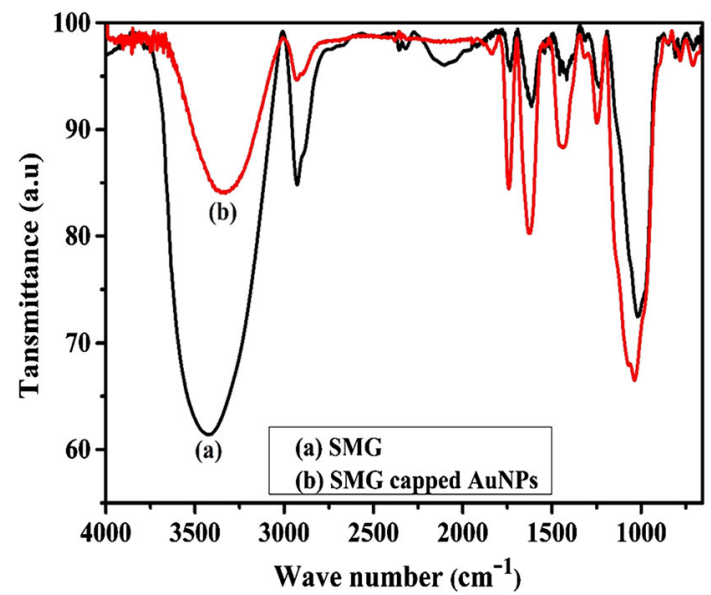

Fig. 3 FTIR spectra of the pure a SMG and b SMG capped AuNPs

assigned to carbonyl stretching vibrations in ketones, aldehydes and carboxylic acids. The sharp band found at $1629 \mathrm{~cm}^{-1}$ could be assigned to characteristic asymmetrical stretch of carboxylate group. The stronger band found at $1427 \mathrm{~cm}^{-1}$ could be assigned to characteristic bending of $-\mathrm{C}-\mathrm{H}$ group. The peak at $1240 \mathrm{~cm}^{-1}$ was due to the $\mathrm{C}-$ $\mathrm{O}$ stretching vibrations of polyols and alcoholic groups. While the IR spectrum of SMG capped AuNPs showed (Fig. 3b) characteristic absorbance bands at 3332, 2927, 2123, 1842, 1741, 1627, 1439, 1242 and $1029 \mathrm{~cm}^{-1}$, respectively, in the IR spectrum of nanoparticles, a shift in the absorbance peaks was observed from 3421 to 3332 , 1729-1741, 1629-1627 and 1427-1439 $\mathrm{cm}^{-1}$. The extra shoulder peak observed at $1830 \mathrm{~cm}^{-1}$ indicates the attachment of $\mathrm{COO}^{-}$to the surface of the AuNPs. FTIR spectral studies suggest that the carbonyl and hydroxyl groups have a stronger affinity to bind with metal and facilitate the formation of a coat over the nanoparticles and favour in stabilizing the AuNPs against agglomeration.

\section{XRD}

The crystalline nature of green-synthesized AuNPs was confirmed by XRD analysis. Diffraction peaks were observed at $38.21^{\circ}, 44.24^{\circ}, 64.31^{\circ}$ and $77.46^{\circ}$ (Fig. 4) which can be indexed as (111), (200), (220) and (311), respectively, and the planes of face-centred cubic (fcc) AuNPs. The existence of diffraction peaks were matched to the standard data files (the JCPDS card No. 04-0784) for all reflections. No extra peaks were found in XRD-spectrum, indicating the synthesized AuNPs were purely crystalline. Crystallite size of AuNPs was determined using the Scherer's formula from the XRD pattern and was found to be around $13.2 \mathrm{~nm}$. The observations from the XRD analysis can very well be correlated with the values obtained from TEM images. 


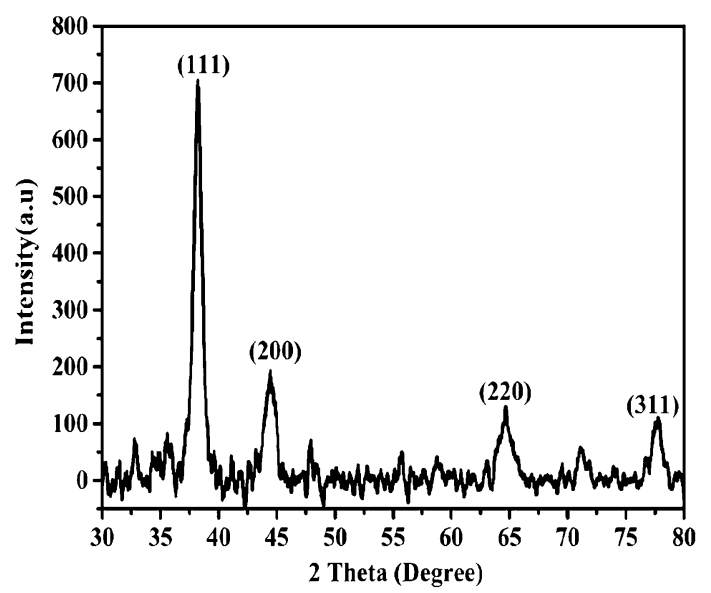

Fig. 4 XRD pattern of gold nanoparticles stabilized by gum SMG. Conditions: $1 \%(\mathrm{w} / \mathrm{v})$ gum SMG, $1 \mathrm{mM} \mathrm{HAuCl}_{4}$ autoclaved for $15 \mathrm{~min}$ at $15 \mathrm{psi}$

\section{$E D X$}

The green synthesis of AuNPs by SMG was further characterized by EDX analysis, which gives the additional evidence for the reduction of $\mathrm{HAuCl}_{4}$ to elemental gold. The synthesized nanoparticles showed a strong peak of $\mathrm{Au}$ along with a weak carbon and oxygen peaks, which may originate from the gum that were bound to the surface of the AuNPs (Fig. 5). The peak of $\mathrm{Cu}$ is also observed which could originate from the carbon coated $\mathrm{Cu}$ grid.

\section{TEM}

The size, shape, morphology and distribution of the AuNPs were analysed using TEM. The TEM images (Fig. 6a, b) shows that the particles are predominantly spherical. The spherical nanoparticles were formed with diameters ranging from 5 to $20 \mathrm{~nm}$ and are of highly mono-dispersed in nature. The average particle size was found to be $12 \pm 2 \mathrm{~nm}$ and the particle size distribution histogram (Fig. 6c) was constructed by counting the size of 150 particles. The selected-area electron diffraction pattern was shown in Fig. 6d.

\section{Catalytic activity of gold nanoparticles}

\section{Catalytic degradation of methylene blue}

A potential application of synthesized AuNP catalytic activity was the reduction of aqueous MB to Leuco MB in the presence of excess $\mathrm{NaBH}_{4}$. The reaction was monitored by UV-Vis spectrophotometry in the wavelength range between 450 and $750 \mathrm{~nm}$ at room temperature. In aqueous medium, MB shows the absorption peaks at 664 and
Fig. 5 EDX analysis of gold nanoparticles synthesized with $1 \%(\mathrm{w} / \mathrm{v})$ gum SMG and $1 \mathrm{mM}$ $\mathrm{HAuCl}_{4}$, autoclaved for $15 \mathrm{~min}$ at $15 \mathrm{psi}$
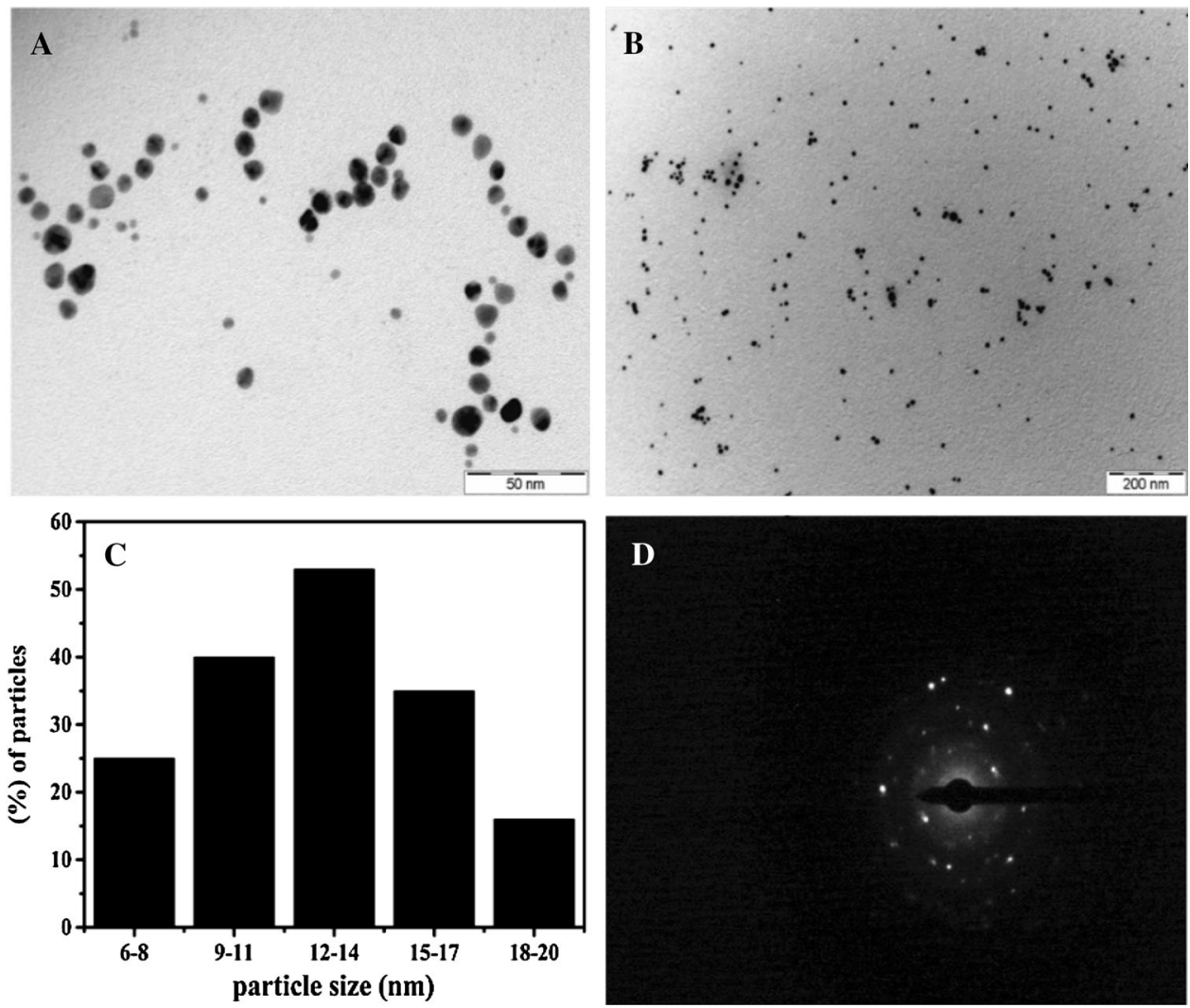
Fig. 6 TEM images of gold nanoparticles synthesized with $1 \%(\mathrm{w} / \mathrm{v})$ gum SMG and $1 \mathrm{mM}$ $\mathrm{HAuCl}_{4}$, autoclaved for $15 \mathrm{~min}$, at 15 psi. a $50 \mathrm{~nm}$ scale, b $200 \mathrm{~nm}$ scale, c the corresponding size distribution histogram and $\mathbf{d}$ corresponding SAED pattern

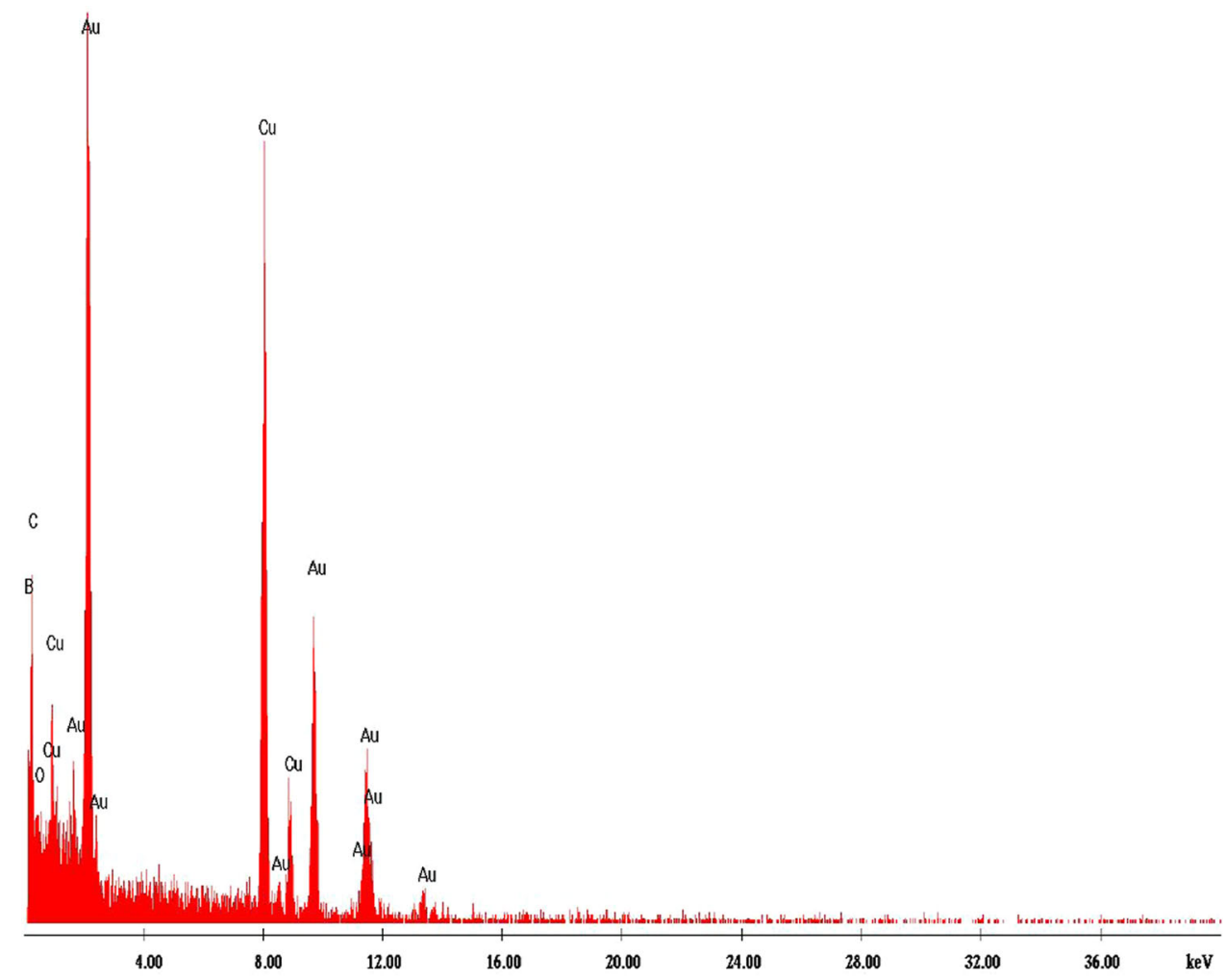

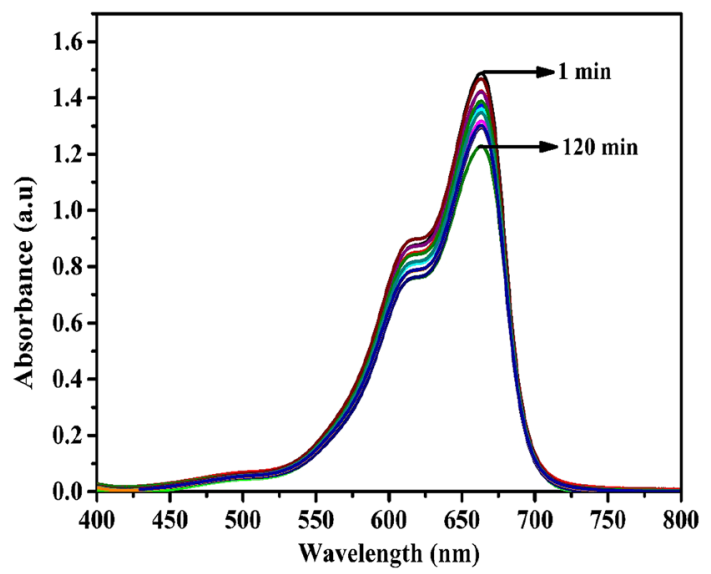

Fig. 7 Reduction of methylene blue dye in the presence of $\mathrm{NaBH}_{4}$ and absence of AuNPs

$614 \mathrm{~nm}$ [20]. Figure 7 shows the reduction of $\mathrm{MB}$ by $\mathrm{NaBH}_{4}$ in the absence of gold nano catalyst for a time period of $120 \mathrm{~min}$. A small decreasing trend of the absorption maximum indicates the reduction of $\mathrm{MB}$, but in a slow pace. The UV-Vis spectrum of the reduction of MB by $\mathrm{NaBH}_{4}$ in the presence of catalytically active AuNPs is shown in Fig. 8. The reduction process was found to be accelerated in the presence of gold nano colloids which showed a rapid decrease in the absorption intensity of MB solution. AuNPs help in the electron relay from $\mathrm{BH}_{4}^{-}$ (donor) to $\mathrm{MB}$ (acceptor). $\mathrm{BH}_{4}^{-}$ions are nucleophilic, while

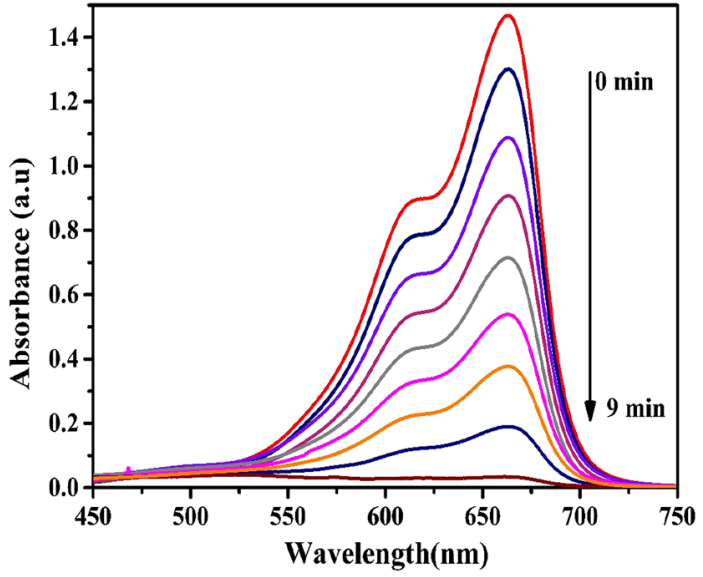

Fig. 8 Time-dependent UV-Vis spectra for the catalytic reduction of methylene blue to leuco methylene blue by $\mathrm{NaBH}_{4}$ in the presence of AuNPs. Conditions: $[\mathrm{MB}]=1 \mathrm{mM} ; \quad\left[\mathrm{NaBH}_{4}\right]=10 \mathrm{mM}$; gold nanoparticles obtained from $1 \mathrm{mM}$ of chloroauric acid and $1 \%$ of gum SMG

MB are electrophilic in nature with respect to AuNPs, where the AuNPs accept electrons from $\mathrm{BH}_{4}^{-}$ions and conveys them to the MB (Fig. 9) [21]. The absorption spectrum showed the decreasing peaks in intensity for $\mathrm{MB}$ dye at different time intervals. Initially, the absorption peak at $664 \mathrm{~nm}$ for MB dye was found to decrease only gradually with the increase in the reaction time indicating that the dye has been degraded slowly [22]. The linear 
<smiles>CN(C)C1=CC2Sc3cc(N(C)C)ccc3N([TlH])C3C=CC(N(C)C)=CC3C2C=C1</smiles>

Fig. 9 Reduction reaction of MB to LMB

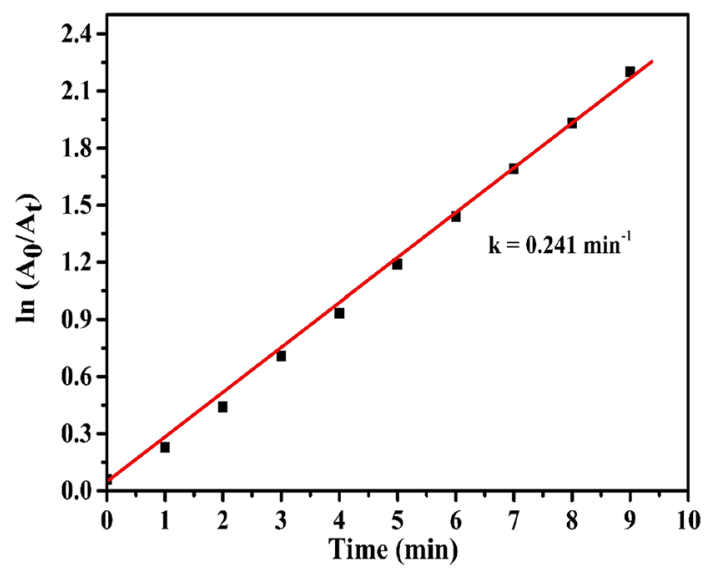

Fig. 10 The plot of $\ln \left(A_{0} / A_{t}\right)$ versus time for the reduction of methylene blue

correlation between $\ln \left(A_{t} / A_{0}\right)$ versus reduction time in minutes (Fig. 10) indicates that the reduction follows a pseudo-first-order reaction kinetics with respect to $\mathrm{MB}$ as the concentration of $\mathrm{NaBH}_{4}(10 \mathrm{mM})$, was relatively larger than that of $\mathrm{MB}(1 \mathrm{mM})$. The rate constant is calculated from the slope of this graph and is found to be $0.241 \mathrm{~min}^{-1}$.

\section{Catalytic degradation of congo red}

The prepared AuNPs could be effectively used to catalyse the decolourization of CR, a kind of azo dye with two $\mathrm{N}=\mathrm{N}-$ bonds as shown in Fig. 11. It is an anionic dye widely used in textiles, paper, plastic and rubber industries.

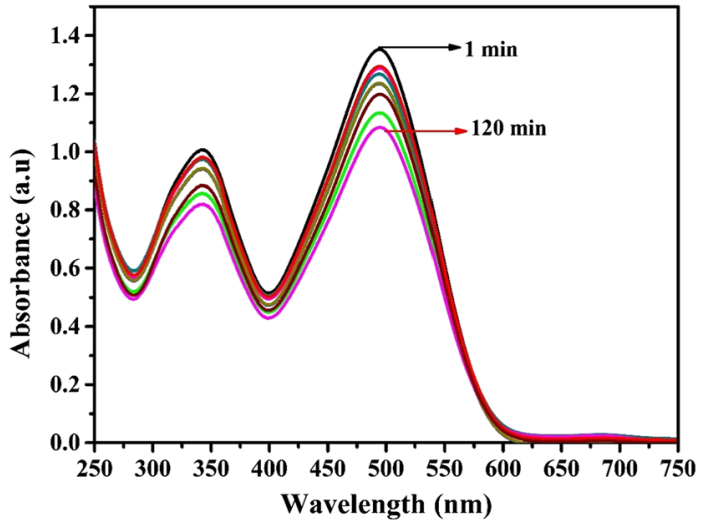

Fig. 12 Reduction of Congo red dye in the presence of $\mathrm{NaBH}_{4}$ and absence of AuNPs

The reaction was monitored by $\mathrm{UV}-\mathrm{Vis}$ spectrometry in the wavelength range between 250 to $700 \mathrm{~nm}$ at room temperature. In aqueous medium, CR shows an absorption band at $498 \mathrm{~nm}\left(\pi \rightarrow \pi^{*}\right)$ and $350 \mathrm{~nm}\left(n \rightarrow \pi^{*}\right)$, transition associated with the azo group [23]. AuNPs act as an electron relay, and electron transfer take place via AuNPs from $\mathrm{BH}_{4}^{-}$(donor) to $\mathrm{CR}$ (acceptor) molecules. In spite of adding $\mathrm{NaBH}_{4}$ to dye solutions, no considerable colour change was observed for a long time. Figure 12 suggests that the reduction of $\mathrm{CR}$ proceeded very slowly in the presence of the strong reducing agent $\mathrm{NaBH}_{4}$ [24]. On the other hand, after the addition of the AuNPs to a mixture of dye and $\mathrm{BH}_{4}^{-}$ions, the reaction mixture was swiftly decolored indicating the remarkable catalytic effect of

Fig. 11 Structure of Congo red
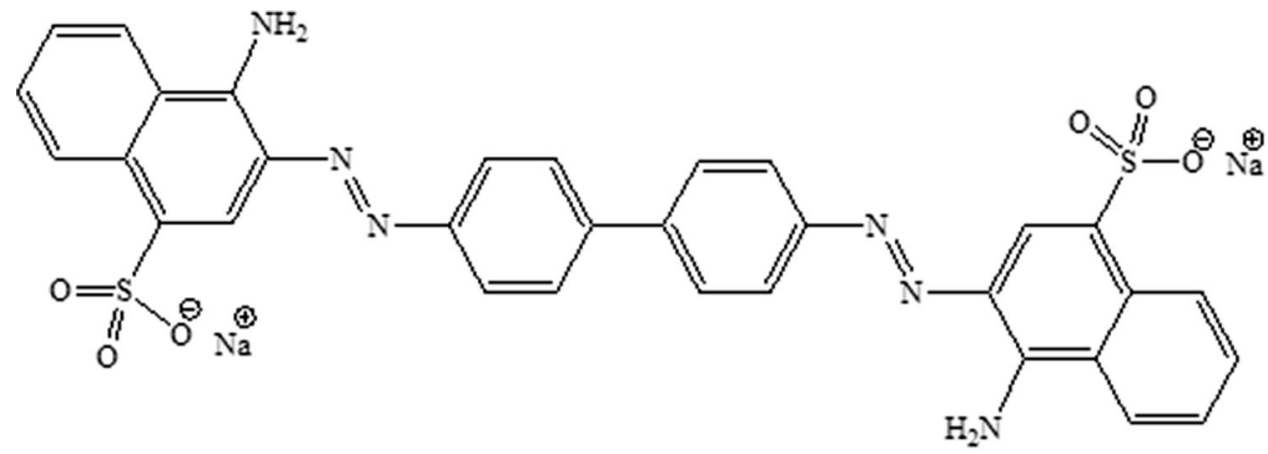


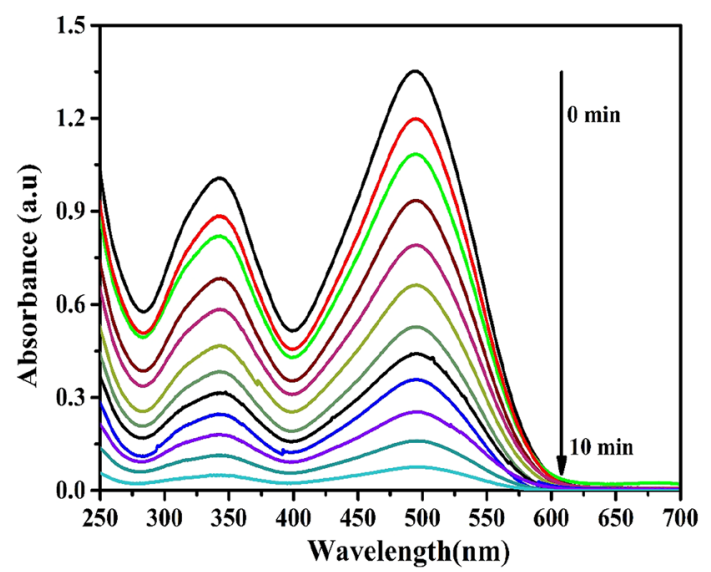

Fig. 13 Time-dependent UV-Vis spectra for the catalytic reduction of Congo red by $\mathrm{NaBH}_{4}$ in the presence of AuNPs. Conditions: $[\mathrm{CR}]=1 \mathrm{mM} ; \quad\left[\mathrm{NaBH}_{4}\right]=10 \mathrm{mM} ;$ gold nanoparticles obtained from $1 \mathrm{mM}$ of chloroauric acid and $1 \%$ of gum SMG

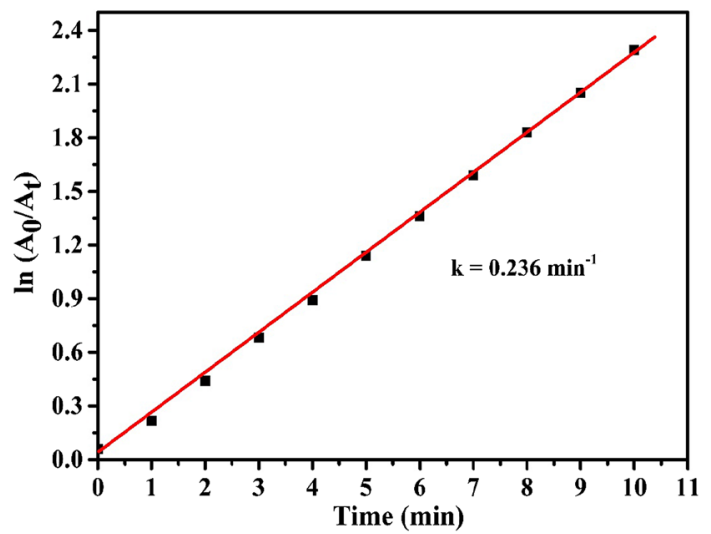

Fig. 14 The plot of $\ln \left(A_{0} / A_{t}\right)$ versus time for the reduction of CR

AuNPs in the degradation of CR. Figure 13 shows the UVVis absorption spectrum of CR dye showing a gradual decrease in peak intensity due to the reduction by $\mathrm{NaBH}_{4}$ in the presence of AuNP catalyst. The rate constant $(k)$ was determined from the linear plot of $\ln \left(A_{0} / A_{t}\right)$ versus reduction time in minutes (Fig. 14). The degradation reaction follows a pseudo first order reaction kinetics with respect to CR because the concentration of $\mathrm{NaBH}_{4}(10 \mathrm{mM})$, is larger than that of $\mathrm{CR}(1 \mathrm{mM})$. The reaction rate constant was calculated and was found to be $0.236 \mathrm{~min}^{-1}$.

\section{Conclusion}

A facile, non-hazardous, cost-effective and a green approach for the synthesis of AuNPs was developed through the reduction of aqueous $\mathrm{HAuCl}_{4}$ solution using SMG as reducing and stabilizing agent. The synthesized nanoparticles were characterized by XRD which confirmed the face-centred cubic crystalline phase. TEM results revealed that the average size of synthesized AuNPs was around $12 \pm 2 \mathrm{~nm}$. The examination of AuNPs by UV-Vis spectroscopy demonstrates that the AuNPs formed are nanosized and the absorption peak range is $520-530 \mathrm{~nm}$. The hydroxyl functional groups present in the gum were found to be responsible for the formation of AuNPs. The green-synthesized AuNPs were proven as efficient catalysts with enhanced rates of reduction of $\mathrm{MB}$ and $\mathrm{CR}$ dyes.

Acknowledgments One of the authors Bhagavanth Reddy G gratefully acknowledges CSIR, New Delhi, for providing senior research fellowship.

Open Access This article is distributed under the terms of the Creative Commons Attribution 4.0 International License (http://crea tivecommons.org/licenses/by/4.0/), which permits unrestricted use, distribution, and reproduction in any medium, provided you give appropriate credit to the original author(s) and the source, provide a link to the Creative Commons license, and indicate if changes were made.

\section{References}

1. Rosarin, F.S., Mirunalini, S.: Nobel metallic nanoparticles with novel biomedical properties. J. Bioanal. Biomed. 03, 85-91 (2011)

2. Dhar, S., Reddy, E.M., Shiras, A., Pokharkar, V., Prasad, B.L.V.: Natural gum reduced/stabilized gold nanoparticles for drug delivery formulations. Chem. Eur. J. 14, 10244-10250 (2008)

3. Patel, A., Prajapati, P., Boghra, R.: Overview on application of nanoparticles. ajpscr 1, 40-55 (2011)

4. El-Brolossy, T., Abdallah, T., Mohamed, M.B., Abdallah, S., Easawi, K., Negm, S., Talaat, H.: Shape and size dependence of the surface plasmon resonance of gold nanoparticles studied by photoacoustic technique. Eur. Phys. J. Spec. Top. 153, 361-364 (2008)

5. Hashmi, S.K., Hutchings, G.J.: Gold catalysis. Angew. Chem. Int. Ed. Engl. 45, 7896-7936 (2006)

6. Farhadi, K., Forough, M., Molaei, R., Hajizadeh, S., Rafipour, A.: Highly selective $\mathrm{Hg}^{2+}$ colorimetric sensor using green synthesized and unmodified silver nanoparticles. Sensors Actuators B Chem. 161, 880-885 (2012)

7. Kumar, A., Zhang, X., Liang, X.: Gold nanoparticles: emerging paradigm for targeted drug delivery system. Biotechnol. Adv. 31, 593-606 (2013)

8. Hameed, B.H., Ahmad, L., Latiff, K.N.: Adsorption of basic dye (methylene blue) onto activated carbon prepared from rattan sawdust. Dye. Pigment. 75, 143-149 (2007)

9. Wanyonyi, W.C., Onyari, J.M., Shiundu, P.M.: Adsorption of congo red dye from aqueous solutions using roots of eichhornia crassipes: kinetic and equilibrium studies. Energy Proc. 50, 862-869 (2014)

10. Narband, N., Uppal, M., Dunnill, C.W., Hyett, G., Wilson, M., Parkin, I.P.: The interaction between gold nanoparticles and cationic and anionic dyes: enhanced UV-visible absorption. Phys. Chem. Chem. Phys. 11, 10513-10518 (2009)

11. Ashokkumar, S., Ravi, S., Kathiravan, V., Velmurugan, S.: Synthesis, characterization and catalytic activity of silver nanoparticles usingTribulus terrestrisleaf extract. Spectrochim. Acta Part A Mol. Biomol. Spectrosc. 121, 88-93 (2014)

12. Jagtap, N.R., Shelke, V., Nimase, M.S., Jadhav, S.M., Shankarwar, S.G., Chondhekar, T.K.: Electrochemical synthesis of tetra 
alkyl ammonium salt stabilized gold nanoparticles. Synth. React. Inorg. Met. Nano-Metal Chem. 42, 1369-1374 (2012)

13. Eustis, S., Hsu, H., El-sayed, M.A.: Gold nanoparticle formation from photochemical reduction of $\mathrm{Au}^{3+}$ by continuous excitation in colloidal solutions. A proposed molecular mechanism. J. Phys. Chem. B 109, 4811-4815 (2005)

14. Punuri, J., Sharma, P., Sibyala, S., Tamuli, R., Bora, U.: Piper betle-mediated green synthesis of biocompatible gold nanoparticles. Int. Nano Lett. 2, 18 (2012)

15. Majumdar, R., Bag, B.G., Maity, N.: Acacia nilotica (Babool) leaf extract mediated size-controlled rapid synthesis of gold nanoparticles and study of its catalytic activity. Int. Nano Lett. (2013). doi:10.1186/2228-5326-3-53

16. Das, S., Ghosal, P.K., Ray, B.: Note structural studies of a polysaccharide salmalia malabarica. Carbohydr. Res. 207, 336-339 (1990)

17. De, D., Ali, K.M., Chatterjee, K., Bera, T.K., Ghosh, D.: Antihyperglycemic and antihyperlipidemic effects of $N$-hexane fraction from the hydro-methanolic extract of sepals of salmalia malabarica in streptozotocin-induced diabetic rats. J. Complement. Integr. Med. (2012). doi:10.1515/1553-3840.1565

18. Faizi, S., ur-Rehman, S.Z., Naz, A., Muhammad, A.V., Dar, A., Naqv, S.: bioassay-guided studies onBombaxceiba leaf extract: isolation of shamimoside, a new antioxidant xanthonecglucoside. Chem. Nat. Compd. 48, 774-779 (2012)
19. Martínez, J.C., Chequer, N.A., González, J.L., Cordova, T.: Alternative metodology for gold nanoparticles diameter characterization using PCA technique and UV-Vis spectrophotometry. Nanosci. Nanotechnol. (2013). doi:10.5923/j.nn.20120206.06

20. Uddin, M.J., Islam, A., Haque, S.A., Hasan, S.: Preparation of nanostructured $\mathrm{TiO}_{2}$-based photocatalyst by controlling the calcining temperature and $\mathrm{pH}$. Int. Nano Lett. (2012). doi:10.1186/ 2228-5326-2-19

21. Cheval, N., Gindy, N., Flowkes, C., Fahmi, A.: Polyamide 66 microspheres metallised with in situ synthesised gold nanoparticles for a catalytic application. Nanoscale Res. Lett. (2012). doi:10.1186/1556-276X-7-182

22. Ke, L., Xuegang, L., Xiaoyan, L., Fangwei, Q., Pei, W.: Novel $\mathrm{NiCoMnO}_{4}$ thermocatalyst for low-temperature catalytic degradation of methylene blue. J. Mol. Catal. A: Chem. doi:10.1016/j. molcata.2013.11.017

23. Farzaneh, F., Haghshenas, S.: Facile synthesis and characterization of nanoporous $\mathrm{NiO}$ with folic acid as photodegredation catalyst for congo red. Mater. Sci. Appl. 3, 697-703 (2012)

24. Xu, L., Wu, X.C., Zhu, J.J.: Green preparation and catalytic application of Pd nanoparticles. Nanotechnology (2008). doi:10. 1088/0957-4484/19/30/305603 